\title{
Primitive neuroectodermal tumors in patients with testicular germ cell tumors usually resemble pediatric-type central nervous system embryonal neoplasms and lack chromosome 22 rearrangements
}

\author{
Thomas M Ulbright ${ }^{1}$, Eyas M Hattab ${ }^{1}$, Shaobo Zhang ${ }^{1}$, Yaron Ehrlich ${ }^{2}$, Richard S Foster ${ }^{2}$, \\ Lawrence $\mathrm{H}$ Einhorn ${ }^{3}$ and Liang Cheng ${ }^{1}$ \\ ${ }^{1}$ Departments of Pathology and Laboratory Medicine, Indiana University School of Medicine, Indianapolis, \\ IN, USA; ${ }^{2}$ Department of Urology, Indiana University School of Medicine, Indianapolis, IN, USA and \\ ${ }^{3}$ Department of Medicine, Indiana University School of Medicine, Indianapolis, IN, USA
}

\begin{abstract}
Primitive neuroectodermal tumors (PNETs) are one of the most frequent types of 'non-germ cell' tumor in patients with testicular germ cell tumors and have a guarded prognosis when present in metastatic sites after cisplatin-based chemotherapy. Improved treatments, including targeted therapy, require understanding the biology of these neoplasms. We therefore analyzed the morphologic, immunohistochemical and molecular biologic features of 14 PNETs from 14 patients with concurrent or previous testicular germ cell tumors; 12 tumors were from metastatic sites and 2 were primary in the testis. Using standard light microscopic criteria for central nervous system and peripheral PNETs, we classified nine tumors as medulloepithelioma, three as medulloblastoma/supratentorial PNET, one as neuroblastic tumor with abundant neuropil and true rosettes and one as small cell embryonal tumor/PNET (Ewing sarcoma-like). Immunostains directed against INI1, CD57, S-100 protein, NeuN, WT1, neurofilament, CD99, GFAP, synaptophysin, chromogranin, AE1/AE3 cytokeratin, Fli-1 and collagen IV were performed for each case. INI1 was diffusely and strongly positive in all tumors whereas the other stains, except for cytoplasmic WT1 (which showed substantial reactivity in most tumors), were mostly focal to negative, including CD99 (eight negative, six focal) and Fli-1 (all negative). The most consistently reactive 'neuroendocrine' marker was CD57. Each case was also analyzed for chromosome 22 rearrangements using a FISH-based break-apart probe method. Only 1 tumor, classified as medulloepithelioma, was scored positive for chromosome 22 translocation (22\% rearranged cells) and the remaining 13 were negative, including the one case that resembled peripheral PNET. We conclude that PNETs derived from testicular germ cell tumors mostly resemble central nervous system PNETs and generally lack the chromosome 22 translocation of peripheral PNETs. Future treatment strategies should take these findings into account. Modern Pathology (2010) 23, 972-980; doi:10.1038/modpathol.2010.70; published online 26 March 2010
\end{abstract}

Keywords: primitive neuroectodermal tumor; testicular neoplasms; germ cell tumor; immunohistochemistry; FISH; translocation; Ewing sarcoma

The development of a metastatic primitive neuroectodermal tumor (PNET) in a patient with a testicular germ cell tumor carries a guarded prognosis. Ganjoo

Correspondence: TM Ulbright, MD, Department of Pathology and Laboratory Medicine, Clarian Pathology Laboratory, Room 4014 350 W. 11th Street, Indianapolis, IN 46202, USA.

E-mail: tulbrigh@iupui.edu

Received 26 October 2009; revised 18 January 2010; accepted 19 January 2010; published online 26 March 2010 et $a l^{1}$ reported that only $44 \%$ of patients who presented with metastases were disease free at a median follow-up of 19 months. Although newer chemotherapy protocols show potential for improving this outcome, there remains a need for more effective treatment. ${ }^{2}$

Targeted modalities have proved efficacious in a number of tumor types, either with specific genetic changes that activate certain cellular signaling pathways or that lack mutations capable of bypassing 
an inhibitory effect. Thus, imatinib mesylate is useful in the treatment of gastrointestinal stromal tumors with specific activating mutations in the KIT gene; ${ }^{3}$ trastuzumab frequently induces clinical responses in breast carcinomas with ERBB2 amplification, ${ }^{4}$ and cetuximab is only effective in colorectal carcinomas with wild-type KRAS. ${ }^{5}$ Although targeted therapy is not yet available for germ cell tumor-derived PNETs, it makes sense to analyze the nature of these neoplasms from their histomorphological, immunohistochemical and genetic aspects in anticipation of the application of targeted therapy at some future time. This is particularly the case with PNET, because the term 'PNET' is applied to several tumor types, notably a family of central nervous system neoplasms that occur mostly in children and which have an embryonal-type morphology (central PNET), ${ }^{6-8}$ as well as to a more homogenous entity-Ewing sarcoma/PNET-that is caused by a specific chromosomal translocation $(t(11 ; 22))$ (peripheral PNET). ${ }^{9,10}$ The former entity includes neoplasms such as medulloblastoma, supratentorial PNET, medulloepithelioma and others. We therefore report the results of a light microscopic, immunohistochemical and molecular biologic investigation of 14 PNETs from patients with testicular germ cell tumors.

\section{Materials and methods}

This study was approved by the Institutional Review Board of Indiana University Purdue University at Indianapolis. A computerized text search of surgical pathology reports at Indiana University Hospital was performed for the phrase 'primitive neuroectodermal.' The reports were reviewed to determine if the diagnosis represented PNETs, if the patient was a testicular germ cell tumor patient, and if the case was 'in house' with available paraffin blocks of tumor. In this way, 12 metastatic PNETs (10 retroperitoneal, 1 liver and 1 kidney) from 12 testicular germ cell tumor patients were identified. In addition, two primary testicular PNETs that were associated with other germ cell tumor types were identified in two separate patients. None of the cases were included in a previous study of germ cell tumor-related PNETs from our institution. ${ }^{11}$ The hematoxylin and eosin slides of these cases were reviewed to allow selection of appropriate blocks for immunohistochemical and molecular studies.

For immunohistochemical studies, four micron, formalin-fixed, paraffin-embedded tissue sections were picked-up and dried on positively charged slides (Superfrost Plus, Erie Scientific, Portsmouth, NH, USA), deparaffinized in xylene and rehydrated through graded alcohols to distilled water. The antibody sources, dilutions and antigen retrieval methodologies are shown in Table 1. The detection method varied by antibody. For AE1/AE3 anticytokeratin and antibodies directed against synaptophysin, chromogranin, S-100 protein, NeuN, collagen IV, laminin, CD99, CD57 and Fli-1, a peroxidaseconjugated streptavidin methodology (Labeled Streptavidin-Biotin (LSAB) 2 system, Dako, Carpinteria, CA, USA) with either biotinylated goat anti-rabbit or biotinylated goat anti-mouse secondary antibodies (Dako) was used. For antibodies directed against INI1 and WT1 proteins, the LSAB + (Dako) method was used. For GFAP, a polymer-based method (Ultra Vision One, Lab Vision, Freemont, CA, USA) was used.

The immunohistochemical stains were evaluated on the basis of the extent and intensity of the colorimetric reaction. For extent, $0=$ negative staining; $1=\leq 10 \% ; 2=>10-50 \% ; 3=>50 \%$. For intensity, $1=$ faint; $2=$ moderate; $3=$ strong. Each stain was 'scored' based on extent and intensity by multiplying the two values to achieve results from 0 to 9 .

For fluorescence in situ hybridization, tissue sections $4 \mu \mathrm{m}$ in thickness were prepared from buffered, formalin-fixed, paraffin- embedded tissue blocks containing tumor. The slides were deparaffinized with two washes of xylene, $15 \mathrm{~min}$ each, and

Table 1 Antibody sources and methods

\begin{tabular}{|c|c|c|c|}
\hline Antibody & Source & Dilution & Retrieval method \\
\hline AE1/AE3 & Dako (Carpinteria, CA, USA) & $1: 80$ & Dako Target Retrieval Method, pH 6.0 \\
\hline CD99 (HBA.71) & Dako & Prediluted & Dako Target Retrieval Method, pH 6.0 \\
\hline Synaptophysin & Dako & Prediluted & Dako Target Retrieval Method, pH 6.0 \\
\hline Chromogranin & Dako & Prediluted & None \\
\hline INI1 & $\begin{array}{l}\text { Becton-Dickinson Biosciences } \\
\text { (San José, CA, USA) }\end{array}$ & $1: 250$ & $\begin{array}{l}\text { Borg Decloaker (Biocare Medical, Concord, } \\
\text { CA, USA) }\end{array}$ \\
\hline Fli-1 (C-19) & $\begin{array}{l}\text { Santa Cruz Biotechnology } \\
\text { (Santa Cruz, CA, USA) }\end{array}$ & $1: 120$ & Dako Target Retrieval Method, pH 6.0 \\
\hline WT1 & Dako & $1: 50$ & Borg Decloaker (Biocare Medical) \\
\hline GFAP & Lab Vision (Fremont, CA, USA) & $1: 150$ & None \\
\hline S-100 & Dako & $1: 1000$ & Dako Target Retrieval Method, pH 6.0 \\
\hline CD57 & Cell Marque (Rocklin, CA, USA) & Prediluted & Dako Target Retrieval Method, pH 6.0 \\
\hline NeuN & Abcam (Cambridge, MA, USA) & $1: 100$ & Dako Target Retrieval Method, pH 6.0 \\
\hline Collagen IV & Dako & Prediluted & Proteinase K \\
\hline Laminin & Dako & $1: 20$ & Proteinase $\mathrm{K}$ \\
\hline
\end{tabular}


subsequently washed twice with absolute ethanol, 10 min each and then air dried in the hood. Next, the slides were treated with $10 \mathrm{mM}$ citric acid ( $\mathrm{pH}$ 6.0) (Zymed, CA, USA) at $95{ }^{\circ} \mathrm{C}$ for $10 \mathrm{~min}$, rinsed in distilled water for $3 \mathrm{~min}$ followed by a wash of $2 \times$ SSC (standard saline citrate) for 5 min. Digestion of the tissue was performed by applying $0.4 \mathrm{ml}$ of pepsin $(5 \mathrm{mg} / \mathrm{ml}$ in $0.1 \mathrm{~N} \mathrm{HCl} / 0.9 \mathrm{NaCl})$ (Sigma, St Louis, MO, USA) at $37^{\circ} \mathrm{C}$ for $40 \mathrm{~min}$. The slides were rinsed with distilled water for $3 \mathrm{~min}$, washed with $2 \times$ SSC for $5 \mathrm{~min}$ and air dried. FISH was performed with LSI EWSR1 dual-color break-apart probe covering 22q12. A $500 \mathrm{~kb}$ probe covering EWSR1 upstream sequences labeled with spectrum orange and an $1100 \mathrm{~kb}$ EWSR1 downstream probe labeled with spectrum green. The probes were from Vysis (Downers Grove, IL, USA) and were diluted with $t$ DenHyb 2 (Insitus, Albuquerque, NM, USA) in a ratio of 1:50. In all, $5 \mu$ l of diluted probe was applied to each slide in reduced light conditions. The slides were then covered with a $22 \times 22 \mathrm{~mm}$ cover slip and sealed with rubber cement. Denaturation was achieved by incubating the slides at $83^{\circ} \mathrm{C}$ for $12 \mathrm{~min}$ in a humidified box and hybridization at $37^{\circ} \mathrm{C}$ overnight. The cover slips were removed and the slides were washed twice with $0.1 \mathrm{XSSC} / 1.5 \mathrm{M}$ urea at $45^{\circ} \mathrm{C}$ (20 min for each), followed by a wash with $2 \times$ SSC for $20 \mathrm{~min}$ and with $2 \times$ SSC $/ 0.1 \% \mathrm{NP}-40$ for $10 \mathrm{~min}$ at $45^{\circ} \mathrm{C}$. The slides were further washed with room temperature $2 \times \mathrm{SSC}$ for $5 \mathrm{~min}$. The slides were air dried and counterstained with $10 \mu \mathrm{l}$ DAPI (Insitus), covered with coverslips and sealed with nail polish. The slides were examined using a Zeiss Axioplan 2 microscope (Zeiss, Gottingen, Germany) with the following filters from Chroma (Chroma, Brattleboro, VT, USA): SP-100 DAPI, FITC MF-101 for Spectrum Green and Gold 31003 for Spectrum Orange. The images were acquired with a CCD camera and analyzed with MetaSystem Isis Software (MetaSystem, Belmont, MA, USA). Five sequential focus stacks with $0.4 \mu \mathrm{m}$ intervals were acquired and then integrated into a single image to reduce thickness-related artifacts. As a positive control, a case of Ewing sarcoma/PNET with a known positive translocation, $(\mathrm{t}(11 ; 22))$, as determined by classic cytogenetic analysis, was run in parallel with the study cases.

The method for in situ hybridization analysis with the Vysis break-apart probe has been previously reported. ${ }^{12-17}$ Briefly, 100 nuclei from a tumor area were counted for probe signals under a fluorescence microscope at $\times 1000$ magnification. Only nonoverlapping tumor nuclei with two red and two green signals were counted. Cells with fused green and red signals or close adjacent green and red signals were considered normal. The cells with one fused signal and separated green and red signals, when the distance between green and red signals was greater than twofold the signal diameter, were considered positive for breakapart. A cut-off value of $20 \%$ of nuclei showing a split signal was considered a positive result for gene rearrangement, as has been previously used. ${ }^{12,14,15}$ The in situ hybridization and analysis were performed twice on each the 14 samples at two different times and using different sample identifiers to verify the results.

\section{Results}

\section{Clinical Findings}

The 14 patients ranged from 19 to 43 years of age (median 30.5 years) at the time of surgical resection of the specimen containing the PNET. The PNET was located in the retroperitoneum in 10 cases, the testis in 2 , and the liver and kidney (1 each). Eleven patients had established non-seminomatous testicular germ cell tumors based on review of the orchiectomy specimens at our institution. Teratoma was found in each of these 11 testicular specimens, yolk sac tumor in 7 , embryonal carcinoma in 5 and seminoma and choriocarcinoma in 1 each. In addition, in nine of these cases, PNET was identified in the testicular specimens based on the criterion of overgrowth of primitive neuroectodermal tissue to the extent that the majority of a low power (4X objective) field was occupied, as has been previously described for the distinction of PNET from 'immature teratoma." ${ }^{18}$ In one case, no review of the testicular primary had been performed but the diagnosis of a non-seminomatous germ cell tumor was supported by an elevated serum alpha-fetoprotein level at the time of orchiectomy. In addition, one patient, a 43-year-old man (Table 2, case 4), had a pure testicular PNET that had overgrown the testis to the extent that residual seminiferous tubules had been totally replaced, therefore not allowing recognition of intratubular germ cell neoplasia. The final patient had a history of testicular germ cell tumor but no specific information was available regarding the precise elements of his tumor. Nine patients received cisplatin-based chemotherapy before resection of the PNET-bearing specimen and five did not (including the two patients whose testicular primaries were analyzed).

\section{Pathologic Findings}

On gross examination the regions corresponding to PNET consisted of fleshy, solid, gray-white tissue. On microscopic examination, the tumors showed varying proportions of several patterns. Primitive, often anastomosing neural tubules were present in nine cases; these consisted of stratified columnar tumor cells arranged in a radial manner around an open central luminal space, with the peripheral aspect of the tubules distinctly set apart by an apparent basement membrane (Figure 1a). A primitive-appearing oval to spindle cell component partially occupied the intertubular spaces in six of these cases (Figure 1a). Mitotic figures were easily 


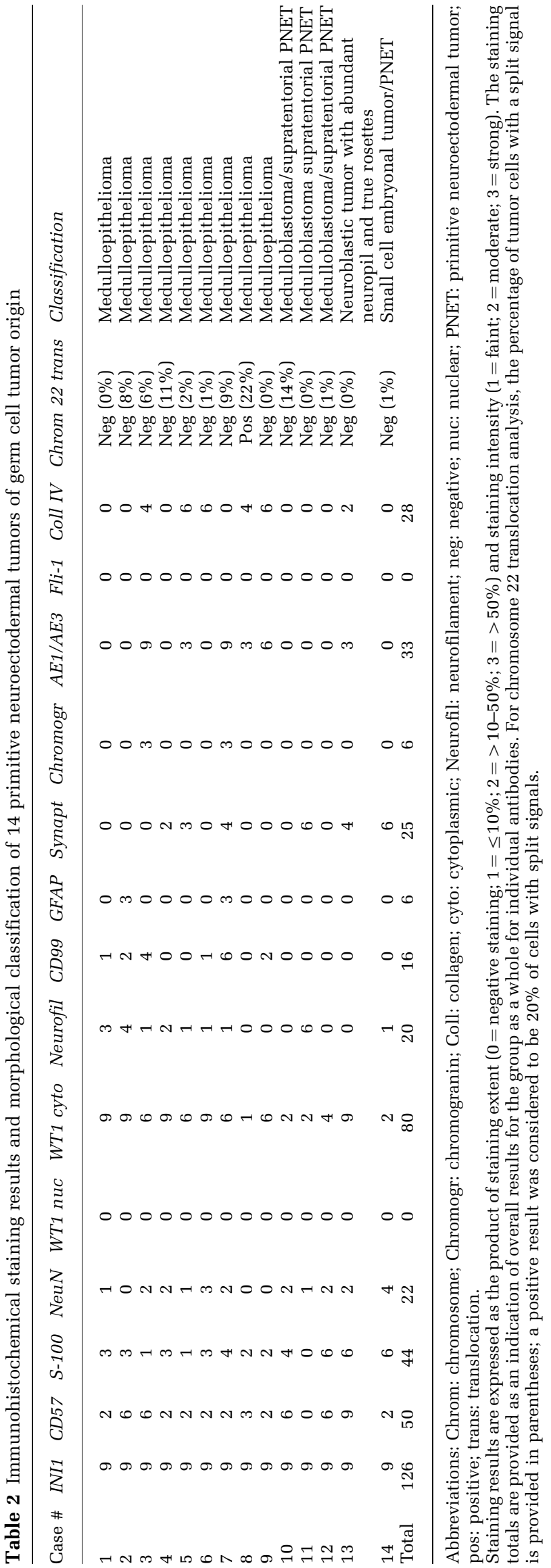

found in the tubules, including along the luminal border. Ganglion cells were identified in the stroma of two and glial cells in one. In accordance with criteria for central nervous system tumors, ${ }^{8}$ such cases were classified as medulloepitheliomas (Table 2). The case of the pure testicular PNET (Table 2, case 4) lacking recognizable germ cell tumor elements of the usual type was among these nine medulloepitheliomas.

Three tumors were classified as medulloblastoma/ supratentorial PNET. The tumor cells were arranged in sheets. Two had cells with scant, ill-defined, pale cytoplasm and indistinct cytoplasmic membranes. In one of these cases, the cells were round and focally associated with a vague, neuropil-like stroma (Figure 1b). In the second of these two tumors, the cells had oval to spindled profiles and formed ill-defined fascicles (Figure 1c). The third example had closely packed but discohesive, polygonal cells with a moderate amount of eosinophilic cytoplasm, prominent nucleoli and frequent mitotic figures and apoptotic bodies (Figure 1d). Distinct rosettes or pseudorosettes were not identified in any of these cases.

One tumor was classified as a neuroblastic tumor with abundant neuropil and true rosettes. ${ }^{19}$ It consisted of primitive-appearing, small to mediumsized cells arranged in clusters in an eosinophilic, fibrillar matrix with occasional true rosettes (Figure 1e).

One final tumor consisted of multiple discrete nests of uniform, round tumor cells in a loose, fibrous stroma (Figure 1f). Mitotic figures were sparse. This case was classified as a small cell embryonal neoplasm, not otherwise specified and was felt to have features reminiscent of peripheral PNET/Ewing sarcoma.

Five tumors showed glomeruloid vascular proliferation. These included two cases each of tumors classified as medulloepithelioma and medulloblastoma/supratentorial PNET and the case showing the features of a neuroblastic tumor with abundant neuropil and true rosettes.

\section{Immunohistochemical Findings}

The immunohistochemical staining results are summarized in Table 2. All tumors stained strongly and diffusely for INI1. A majority of the other markers were patchy and/or weak. Cytoplasmic but not nuclear expression of WT1 occurred in all cases and tended to be more impressive in tumors classified as medulloepithelioma compared with others (Figure 2a), although overall there was no strong correlation between the light microscopic classification and the immunohistochemical findings. Of the markers that are usually considered 'neuroendocrine,' CD57 was most frequently positive (Figure 2b), followed by synaptophysin, NeuN (Figure 2c) and neurofilament (Figure 2d). The latter 

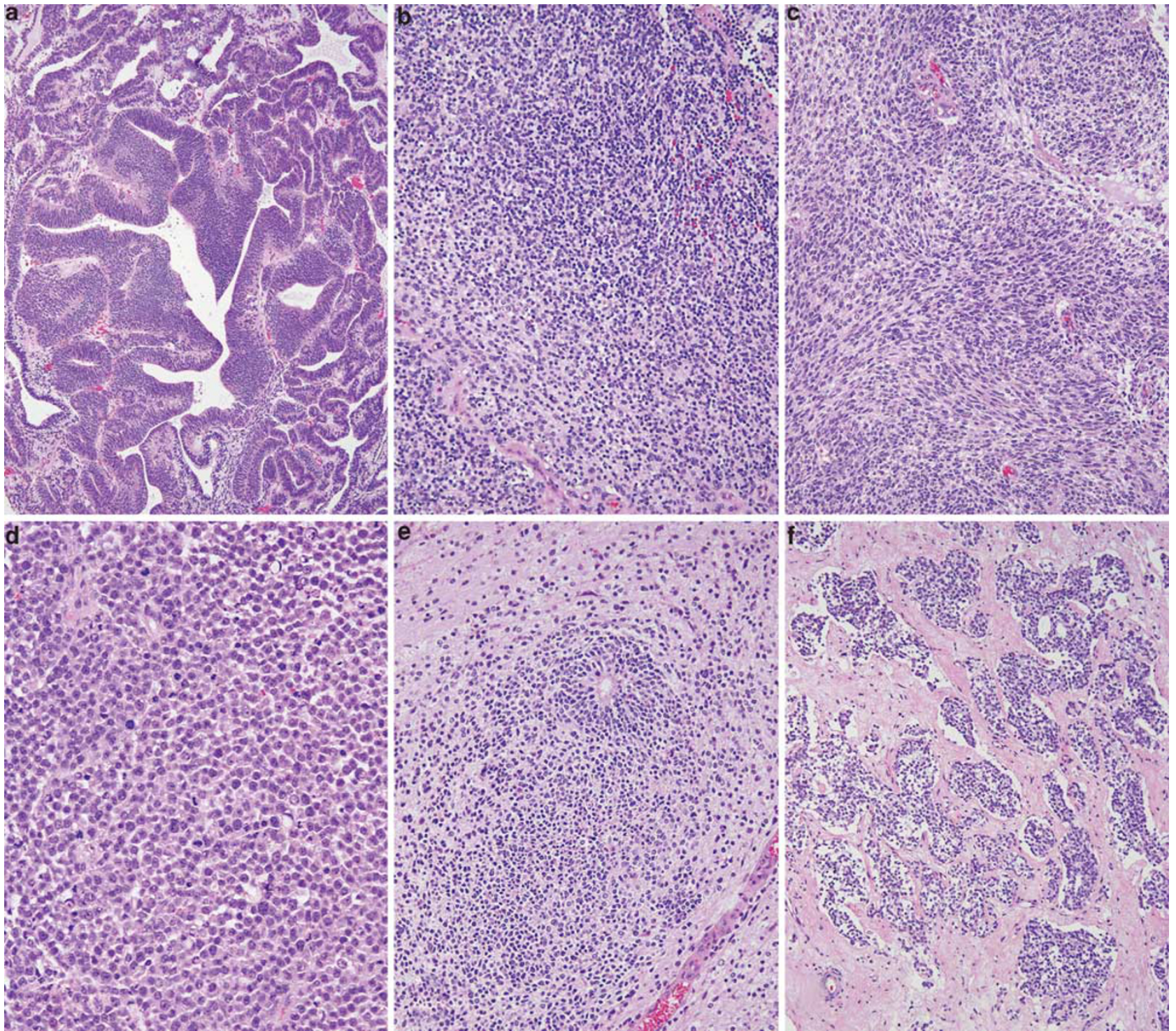

Figure 1 (a) (Case 9) This tumor resembles medulloepithelioma with anastomosing, primitive neural tubules lined by stratified, columnar tumor cells. A neoplastic stromal component is visible at bottom-right. (b) (Case 12) There is a diffuse arrangement of small, primitive tumor cells with a suggestion of neuropil (lower-left) in a tumor classified as medulloblastoma/supratentorial PNET. (c) (Case 10) A medulloblastoma/supratentorial PNET-like tumor has a slightly fascicular arrangement of oval to spindled cells. (d) (Case 11) A tumor classified as medulloblastoma/supratentorial PNET has a diffuse arrangement of moderate sized tumor cells with frequent mitotic figures. (e) (Case 13) This tumor resembles a neuroblastic tumor with abundant neuropil and true rosettes. (f) This tumor resembles peripheral PNET with a nested arrangement of small cells in a fibrous stroma.

three, however, were frequently negative. Chromogranin only marked two cases, both classified as medulloepithelioma. Focal to rare S-100 reactivity occurred in all but one case but was often weak. AE1/AE3 cytokeratin was most likely to be positive in neural tubules and therefore occurred most robustly in a minority of the cases classified as medulloepithelioma (Figure 2e) (Table 2). Collagen IV highlighted the external basement membrane that separated tubular structures from the surrounding stroma in five cases of medulloepithelioma (Figure 2f). GFAP positivity was observed in only two cases, both medulloepitheliomas. The two markers associated with Ewing sarcoma/peripheral
PNET, CD99 and Fli-1, were infrequently reactive (CD99), never strongly and diffusely so, or uniformly negative (Fli-1).

\section{Fluorescence In Situ Hybridization Findings}

Using the chromosome 22 break-apart probe, one case (Table 2, case 8), a testicular primary associated with teratoma, yolk sac tumor and embryonal carcinoma, was scored as positive for chromosome 22 translocation, showing $22 \%$ of tumor nuclei with a positive signal (Table 2). This case was considered to have light microscopic features of 

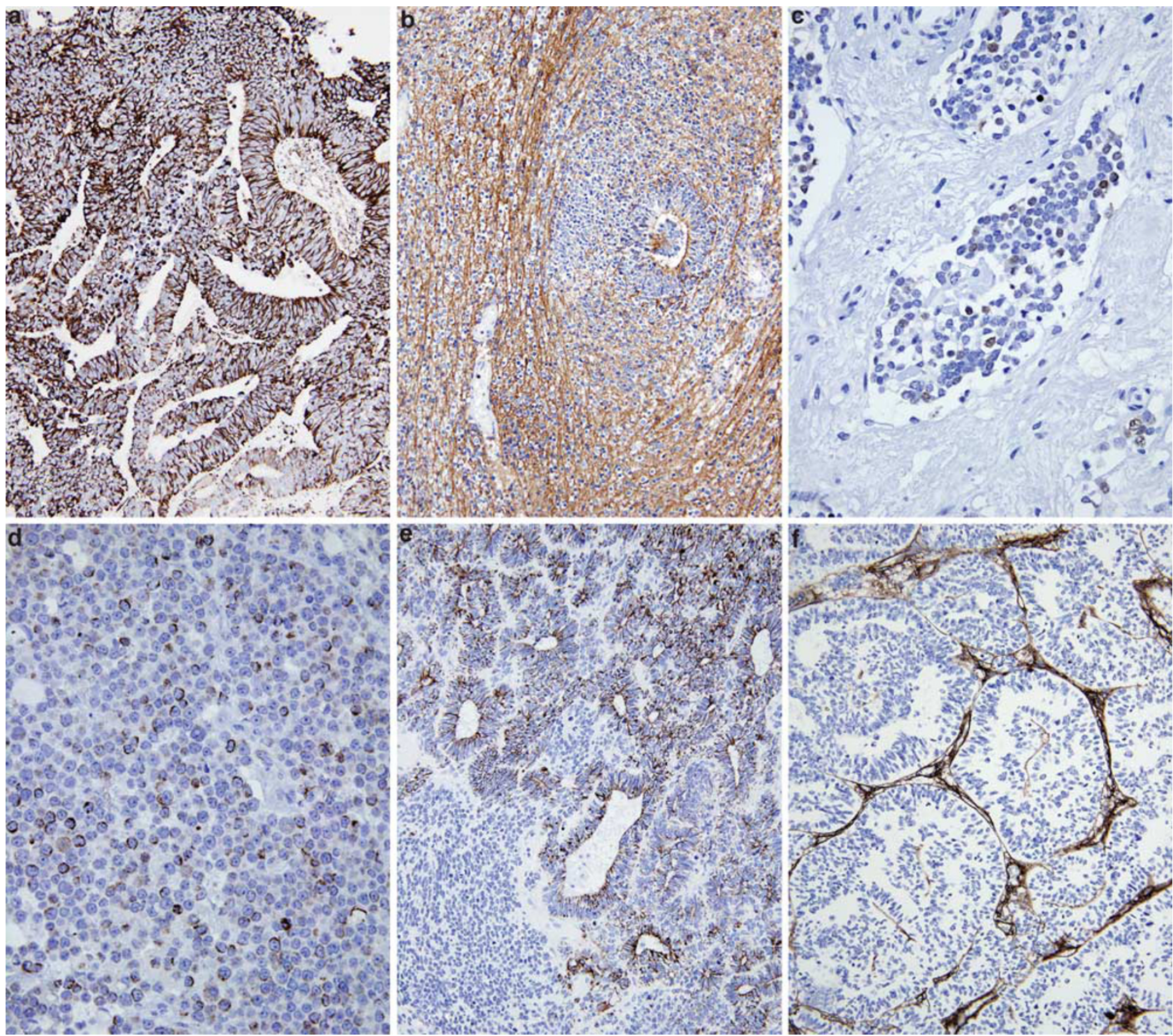

Figure 2 (a) (Case 1) There is strong, diffuse cytoplasmic positivity for WT1 in this tumor resembling medulloepithelioma. (b) (Case 13) The tumor classified as neuroblastic tumor with abundant neuropil and true rosettes shows positivity for CD57. (c) (Case 14) The tumor resembling peripheral PNET shows scattered tumor cells with weak to moderate nuclear reactivity for NeuN. (d) (Case 11) A tumor resembling medulloblastoma/supratentorial PNET shows cytoplasmic reactivity for neurofilament. (e) (Case 7) A tumor resembling medulloepithelioma is positive for AE1/AE3 cytokeratin. (f) (Case 5) An immunostain for collagen IV highlights the basement membrane that outlines the neural tubules of a medulloepithelioma-like tumor.

medulloepithelioma. The immunohistochemical staining pattern of this case (Table 2) overlapped with those of the other tumors. The percentage of cells with positive hybridization signals in the remaining 13 cases fell below this cut-off value, ranging from 0 to $14 \%$ (Table 2).

\section{Discussion}

It has been clear, for over a decade, that PNETs represent one of the more frequent forms of 'nongerm cell' tumor that develop in patients with testicular germ cell tumors and that they have a poor prognosis when found in metastatic sites after primary or secondary chemotherapy. ${ }^{1,11}$ A majority of such PNETs show morphologic features of those primitive tumors of the central nervous system that are characteristically observed in children, ${ }^{11}$ especially in the posterior fossa, and this is also true for the PNETs that develop in the ovary. ${ }^{20}$ There remains, however, relatively scant information regarding the morphologic spectrum of these PNETs. ${ }^{10}$ These considerations prompted this study.

Our study verified a disproportionate frequency of tumors with the morphologic features of 'medulloepithelioma,' which contrasts with the uncommonness of this neoplasm among central nervous system PNETs. ${ }^{8}$ These tumors formed large tubular structures, often with an investing basement 
membrane as shown by collagen IV immunostains, that set the tubules apart from the adjacent stroma. Additional morphologies that are typically encountered in central nervous system neoplasms of children were also found, including neuroblastic tumor with abundant neuropil and true rosettes ${ }^{19,21}$ and medulloblastoma/supratentorial PNET. $^{8}$ One tumor, classified as a small cell embryonal tumor/ PNET, had features that could be observed in either a peripheral PNET/Ewing sarcoma or an undifferentiated embryonal tumor of the central nervous system. We found no evidence for any relationship of our cases to the atypical teratoid/rhabdoid tumor $^{22,23}$ that is mostly observed in the central nervous system of young children in that no distinct rhabdoid morphology was appreciated and there was uniform retention of immunoreactivity for INI1 protein. Our experience was therefore similar to that of Michael et $a l^{11}$ in a similar cohort of testicular germ cell tumor patients. These investigators reported a predominance of testicular PNETs having the morphologic features of medulloepitheliomas, although in that series the metastatic lesions classified as PNETs more commonly resembled neuroblastomas or peripheral PNETs. The same holds true for ovarian PNETs. In the series of Kleinman et $a l,{ }^{20}$ which included 12 cases of PNETs of the ovary among a collection of 25 primary ovarian neuroectodermal tumors, 4 were classified as medulloepitheliomas, 3 as neuroblastomas, 3 as ependymoblastomas and 2 as medulloblastomas.

It is therefore evident that the majority of gonadalderived PNETs do not resemble peripheral PNET/ Ewing sarcoma, but there are some morphologically ambiguous cases. One of the tumors in our series showed a nested arrangement of uniform, small cells in a fibrous stroma in which peripheral PNET/ Ewing sarcoma was a serious diagnostic consideration (case 14, Table 2) (Figure 1f). In the study of Michael et al, ${ }^{11}$ a number of the metastatic tumors of testicular origin were classified as 'peripheral neuroepithelioma' based on their light microscopic appearance and CD99 reactivity, a finding contrasting with this study wherein strong and diffuse CD99 was not encountered, and those tumors that showed some degree of CD99 reactivity did not have morphological features of peripheral PNET/Ewing sarcoma. Nonetheless, these two studies illustrate that peripheral PNET/Ewing sarcoma remains a significant diagnostic consideration for some of the PNETs that occur in testicular germ cell tumor patients.

Our results provide little support that any of the PNETs we encountered in testicular germ cell tumor patients were of the peripheral PNET/Ewing sarcoma type. In a study of 43 cases of PNET/Ewing sarcoma, the combination of immunopositivity for both CD99 and polyclonal Fli-1 (the same antibody used in our study) was considered the best method for confirming the diagnosis, as assessed by analysis of areas under receiver operator curves looking at possible combinations of three markers (monoclonal antibody to Fli-1, polyclonal antibody to Fli-1 and antibody to CD99). ${ }^{14}$ This method had a sensitivity of detection of $93 \%$. In contrast, none of our cases showed positivity for both of these markers and only two had more than $10 \%$ immunoreactivity for CD99, both medulloepitheliomas. One of our cases showed a positive chromosome 22 rearrangement as assessed by a FISH-based break-apart probe assay. In this case, $22 \%$ of the evaluated tumor cells were scored as positive for a separated signal, which was just slightly above our cut-off value of $20 \%$. In other studies, however, of known PNET/Ewing sarcoma that used this same method, the proportion of positive cells was significantly higher. Thus, Bridge et $a 1^{16}$ reported a mean of $79 \%$ positivity (range, $36-96 \%$ ); Yamaguchi et $a 1^{17}$ found $50-90 \%$ positivity; and, using a cut-off value of $30 \%$, all 10 cases of superficial PNET/Ewing sarcoma with interpretable results reported by Terrier-Lacombe et $a l^{13}$ were positive. It is therefore questionable if our single case should be considered a true positive, given the low proportion of tumor cells showing separated probe signals, noting that it would not have been considered positive using the more stringent cutoff of one study. ${ }^{13}$ On the basis of the absence of CD99 and Fli-1 immunoreactivity and its light microscopic appearance, this tumor (Table 2, case 8) is not a peripheral PNET/Ewing sarcoma but a medulloepithelioma-like tumor.

Although most metastatic PNETs in germ cell tumor patients have a distinctive morphology, occasional cases with a diffuse pattern and lacking defined tubules and rosettes raise differential diagnostic considerations that may be observed in the same context. The distinction of central from peripheral PNETs has been the focus of our study, but both nephroblastoma and embryonal rhabdomyosarcoma also merit consideration. In our opinion, nephroblastoma is the most problematic consideration, given its frequent tubular morphology and blastematous component. The tubules in nephroblastoma, however, are typically smaller and are lined by cuboidal, less stratified cells that those in germ cell tumor-related PNETs, which are frequently lined by several layers of columnar cells. The presence of glomeruloid structures and the absence of rosettes and a neurofibrillary-type matrix are also helpful in the recognition of nephroblastoma. Furthermore, nuclear reactivity for WT1 protein occurs in $59-73 \%$ of nephroblastomas ${ }^{24,25}$ but is absent in germ cell tumor-derived PNETs.

Embryonal rhabdomyosarcoma is the most common secondary malignancy in testicular germ cell tumors $^{26,27}$ and may be confused with diffuse PNETs. Occasional distinct rhabdomyoblasts with eosinophilic cytoplasm are helpful in the distinction from PNET, and cross striations are pathognomonic. The complete absence in embryonal rhabdomyosarcoma of tubular structures and 
rosettes is helpful. Appropriate muscle markers (desmin, myogenin) easily permit the distinction of these two entities.

As advances are made in targeted therapy, it is important to understand the molecular basis of neoplasms. Nomenclature can be confusing, both to pathologists and treating physicians. Our study verifies that the tumors that are observed in patients with testicular germ cell tumors and that are commonly classified as PNETs have morphological features akin to pediatric central nervous system tumors of embryonic type. Even those tumors that may show some light microscopic resemblance to peripheral PNET/Ewing sarcoma are, we feel, unlikely to be true peripheral PNETs based on our experience, although appropriate immunohistochemical or molecular evaluation is required. Caution is also urged regarding the interpretation, or better stated, the over-interpretation, of FISH breakapart probe results. In spite of no morphological or immunohistochemical support, one of our cases had a positive FISH assay (verified in duplicate) for chromosome 22 rearrangements at a relatively low cut-off value. We consider this a false-positive result. Therefore, molecular biologic data, while extremely valuable in the understanding and classification of neoplasms, must be interpreted in the context of traditional morphology and immunohistochemistry.

\section{Acknowledgements}

We thank Connie Temms and Lee Ann Baldridge for excellent technical assistance with the immunohistochemical stains. We also thank Tracey Bender for her skilled clerical assistance.

\section{Disclosure/conflict of interest}

The authors declare no conflict of interest.

\section{References}

1 Ganjoo KN, Foster RS, Michael H, et al. Germ cell tumor associated primitive neuroectodermal tumors. J Urol 2001;165:1514-1516.

2 Brames M, Ehrlich Y, Einhorn L. Malignant transformation of teratoma to primitive neuroectodermal tumor (PNET): outcome analysis with PNET based chemotherapy. J Clin Oncol 2009;27(Suppl) Abstr e16121.

3 Heinrich MC, Corless CL, Demetri GD, et al. Kinase mutations and imatinib response in patients with metastatic gastrointestinal stromal tumor. J Clin Oncol 2003;21:4342-4349.

4 Vogel CL, Cobleigh MA, Tripathy D, et al. Efficacy and safety of trastuzumab as a single agent in first-line treatment of HER2-overexpressing metastatic breast cancer. J Clin Oncol 2002;20:719-726.
5 Heinemann V, Stintzing S, Kirchner T, et al. Clinical relevance of EGFR- and KRAS-status in colorectal cancer patients treated with monoclonal antibodies directed against the EGFR. Cancer Treatment Rev 2009;35:262-271.

6 Rogers HA, Miller S, Lowe J, et al. An investigation of WNT pathway activation and association with survival in central nervous system primitive neuroectodermal tumours (CNS PNET). Br J Cancer 2009;100: 1292-1302.

7 Visee S, Soltner C, Rialland X, et al. Supratentorial primitive neuroectodermal tumours of the brain: multidirectional differentiation does not influence prognosis. A clinicopathological report of 18 patients. Histopathology 2005;46:403-412.

8 Kleihues P, Cavenee WK, eds. Pathology and Genetics of Tumours of the Nervous System. IARC Press: Lyon, France, 2000, pp 314.

9 Ludwig JA. Ewing sarcoma: historical perspectives, current state-of-the-art, and opportunities for targeted therapy in the future. Curr Opin Oncol 2008;20: 412-418.

10 Delattre O, Zucman J, Melot T, et al. The Ewing family of tumors-a subgroup of small-round-cell tumors defined by specific chimeric transcripts. $\mathrm{N}$ Engl J Med 1994;331:294-299.

11 Michael H, Hull MT, Ulbright TM, Foster RS, Miller KD. Primitive neuroectodermal tumors arising in testicular germ cell neoplasms. Am J Surg Pathol 1997; 21:896-904.

12 Wang WL, Mayordomo E, Czerniak BA, et al. Fluorescence in situ hybridization is a useful ancillary diagnostic tool for extraskeletal myxoid chondrosarcoma. Mod Pathol 2008;21:1303-1310.

13 Terrier-Lacombe MJ, Guillou L, Chibon F, et al. Superficial primitive Ewing's sarcoma: a clinicopathologic and molecular cytogenetic analysis of 14 cases. Mod Pathol 2009;22:87-94.

14 Mhawech-Fauceglia P, Herrmann F, Penetrante R, et al. Diagnostic utility of FLI-1 monoclonal antibody and dual-colour, break-apart probe fluorescence in situ (FISH) analysis in Ewing's sarcoma/primitive neuroectodermal tumour (EWS/PNET). A comparative study with CD99 and FLI-1 polyclonal antibodies. Histopathology 2006;49:569-575.

15 Jakowski JD, Wakely Jr PE, Jakowski JD, Wakely Jr PE. Cytopathology of extraskeletal myxoid chondrosarcoma: report of 8 cases. Cancer 2007;111:298-305.

16 Bridge RS, Rajaram V, Dehner LP, et al. Molecular diagnosis of Ewing sarcoma/primitive neuroectodermal tumor in routinely processed tissue: a comparison of two FISH strategies and RT-PCR in malignant round cell tumors. Mod Pathol 2006;19:1-8.

17 Yamaguchi U, Hasegawa T, Morimoto Y, et al. A practical approach to the clinical diagnosis of Ewing's sarcoma/primitive neuroectodermal tumour and other small round cell tumours sharing EWS rearrangement using new fluorescence in situ hybridisation probes for EWSR1 on formalin fixed, paraffin wax embedded tissue. J Clin Pathol 2005;58:1051-1056.

18 Ulbright TM, Loehrer PJ, Roth LM, et al. The development of non-germ cell malignancies within germ cell tumors. A clinicopathologic study of 11 cases. Cancer 1984;54:1824-1833.

19 Eberhart CG, Brat DJ, Cohen KJ, Burger PC. Pediatric neuroblastic brain tumors containing abundant neuropil and true rosettes. Pediatr Dev Pathol 2000;3:346-352. 
20 Kleinman GM, Young RH, Scully RE. Primary neuroectodermal tumors of the ovary: a report of 25 cases. Am J Surg Pathol 1993;17:764-778.

21 Fuller C, Fouladi M, Gajjar A, et al. Chromosome 17 abnormalities in pediatric neuroblastic tumor with abundant neuropil and true rosettes. Am J Clin Pathol 2006;126:277-283.

22 Judkins AR, Mauger J, Ht A, et al. Immunohistochemical analysis of hSNF5/INI1 in pediatric CNS neoplasms. Am J Surg Pathol 2004;28:644-650.

23 Burger PC, Yu IT, Tihan T, et al. Atypical teratoid/ rhabdoid tumor of the central nervous system: a highly malignant tumor of infancy and childhood frequently mistaken for medulloblastoma: a Pediatric Oncology Group study. Am J Surg Pathol 1998;22: 1083-1092.
24 Jimenez RE, Folpe AL, Lapham RL, et al. Primary Ewing's sarcoma/primitive neuroectodermal tumor of the kidney: a clinicopathologic and immunohistochemical analysis of 11 cases. Am J Surg Pathol 2002;26:320-327.

25 Ghanem MA, van der Kwast TH, Den Hollander JC, et al. Expression and prognostic value of Wilms' tumor 1 and early growth response 1 proteins in nephroblastoma. Clin Cancer Res 2000;6:4265-4271.

26 Guo CC, Punar M, Contreras AL, et al. Testicular germ cell tumors with sarcomatous components: an analysis of 33 cases. Am J Surg Pathol 2009;33:1173-1178.

27 Malagon HD, Valdez AM, Moran CA, Suster S. Germ cell tumors with sarcomatous components: a clinicopathologic and immunohistochemical study of 46 cases. Am J Surg Pathol 2007;31:1356-1362. 